\title{
Errores en prescripción y transcripción de medicamentos endovenosos en Servicios Pediátricos, Hospital Dr. Hernán Henríquez Aravena. Temuco, 2008-2009
}

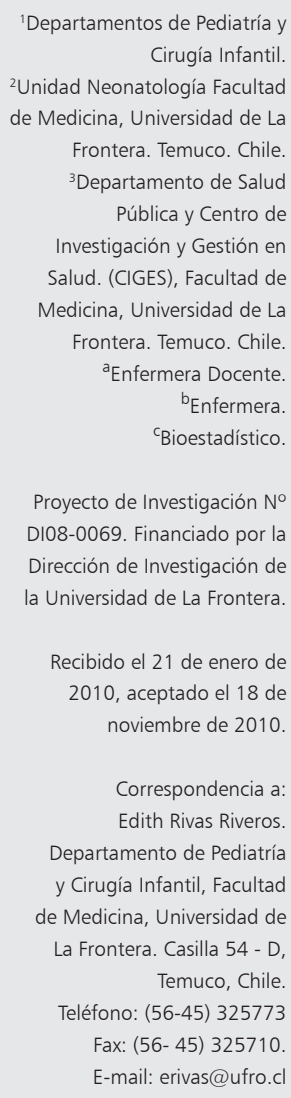

Proyecto de Investigación No DI08-0069. Financiado por la Dirección de Investigación de la Universidad de La Frontera.

Recibido el 21 de enero de 2010, aceptado el 18 de noviembre de 2010

Correspondencia a: Edith Rivas Riveros. Departamento de Pediatría y Cirugía Infantil, Facultad de Medicina, Universidad de La Frontera. Casilla 54 - D, Temuco, Chile. Teléfono: (56-45) 325773 Fax: (56- 45) 325710. E-mail: erivas@ufro.cl

\author{
EDITH RIVAS R. ${ }^{1, a}$, ANGÉLICA RIVAS L., , , LUIS BUSTOS M. ${ }^{3, c}$
}

\section{Frequency of prescription and transcription errors for intravenous medications in four pediatric services}

Background: Errors in the prescription and transcription of medications in pediatric services is a source of adverse events that can be prevented. Aim: To determine and compare the frequency of prescription and transcription errors for intravenous drugs in four pediatric services in a regional general hospital. Material and Methods: Cross sectional analysis of a probabilistic sample of 500 prescriptions of intravenous medications. Information was gathered using an instrument from the American Academy of Pediatrics. Results: The detected prescription errors were illegible indications in $20 \%$, lack of dosing indication in $11 \%$, omission of the administration route in $24 \%$ and omission of the frequency of administration in 15\%. Transcription errors were illegible transcription in 3\%, not transcribing all indicated medications in $4 \%$ and transcription of medications that were not prescribed in $3 \%$. Twenty one percent of prescriptions and 6\% of transcriptions had at least one incorrect action. In the different services studied there was a significant association between the percentage of incorrect prescriptions and transcriptions and between illegible prescriptions and transcriptions. Conclusions: A high percentage of errors in prescription of intravenous medications and their transcriptions, was detected in these services. A better quality of care control is required.

(Rev Med Chile 2010; 138: 1524-1529).

Key words: Drug prescriptions; Errors, medication; Intravenous injections.
$\mathrm{E}$ 1 proceso de medicación es una de las actividades habituales en el tratamiento de pacientes hospitalizados en los servicios pediátricos, cuya cadena de desarrollo implica diversos eslabones, siendo el punto de inicio la prescripción médica. Su ejecución requiere de un entorno facilitador, que permita que el proceso se lleve a cabo de manera eficiente, garantizando la correcta indicación del fármaco a administrar. $\mathrm{Al}$ respecto, errores de medicación son comunes entre pacientes pediátricos y en servicios de emergencia, los cuales pueden conducir a hospitalización prolongada, pruebas de diagnóstico y tratamientos innecesarios e incluso muerte ${ }^{1}$.

A pesar de la importancia creciente de la gestión de riesgos y seguridad del paciente, la evidencia en el ámbito pediátrico internacional y nacional es insuficiente ${ }^{2}$, lo cual ha motivado plantearse el objetivo de determinar la bioseguridad en prescripción y transcripción de medicamentos en- 
dovenosos en servicios pediátricos del Hospital Dr. Hernán Henríquez Aravena, Temuco, 2008-2009, con el propósito de generar estrategias de seguimiento con estándares de calidad que permitan ejecutar el proceso de medicación (prescripción y transcripción) con máxima efectividad.

En prescripción de medicamentos, se analizó legibilidad, vía, dosis y frecuencia de administración, ya que éstas constituyen foco de errores, especialmente en legibilidad, con prescripciones de datos incompletos ${ }^{3}$.

En transcripción de medicamentos, se evaluó: legibilidad, fidelidad, y omisión de fármacos a administrar. En este aspecto, se observó cuidadosamente el cotejo entre el nombre del paciente y su respectiva indicación de medicamentos ${ }^{4}$, como acción previa a la transcripción.

Para identificar acciones incorrectas, se consideró la definición de la National Coordinating Council for Medication Error Reporting and Prevention (NCCMERP): "cualquier incidente que pueda causar daño al paciente o de lugar a una utilización inapropiada de los medicamentos, cuando estos están bajo el control de los profesionales sanitarios o del paciente o consumidor"s. En concordancia, los eventos adversos se relacionan con: la práctica profesional, los productos para el cuidado de la salud, procedimientos y sistemas, (prescripción, comunicación de la prescripción, rótulo del producto, empaque y nomenclatura), composición, distribución; administración; educación de los enfermeros y pacientes, supervisión y uso ${ }^{6}$.

\section{Calidad y Ética en el proceso de medicación}

Calidad y ética son pilares fundamentales que sustentan la medicación, situación que obliga a actuar en consecuencia con la ética de mínimos, la que apunta al desarrollo de acciones básicas que eviten daños al usuario.

Como indicador de calidad se asume efectuar una medicación que asegure entregar al usuario la prestación precisa, para su tratamiento, donde se conjugue la seguridad, efectividad y seguimiento de la terapia medicamentosa. Así, los registros efectuados proporcionan un soporte ético-legal donde quedan consignados los datos, que permitirán detectar o interceptar errores en el sistema ${ }^{7}$.

El estudio se basó en la existencia de acciones incorrectas en la cadena de medicación, con el propósito de generar estrategias de gestión de riesgos que prevengan acontecimientos indeseables.

\section{Objetivos}

a) Establecer porcentajes de error en prescripción y transcripción de medicamentos endovenosos, relacionando ambos aspectos.

b) Comparar porcentajes de error en prescripción y transcripción de medicamentos endovenosos en cuatro servicios pediátricos, Hospital Dr. Hernán Henríquez Aravena, Temuco, 20082009.

\section{Material y Método}

Estudio de corte transversal. Se estimó una muestra de 500 prescripciones para una población de 1.200 niños, considerando un porcentaje de error en éstos de $35,2 \%{ }^{8}$, confianza $95 \%$ y una precisión de 3,2\%. Dicha muestra se subdividió por fijación simple en 125 por cada uno de los cuatro servicios pediátricos, Neonatología, Pediatría, Unidad de Cuidados Intensivos de Pediatría y Cirugía Infantil, debido a la similitud de ingresos y egresos de usuarios.

Datos recolectados a través de fichas clínicas y hojas de enfermería, utilizando la revisión de criterios mínimos establecidos por la American Academy of Pediatrics y la American Society of Health-System Pharmacist, referidos a claridad en las indicaciones médicas en la prescripción de medicamentos y su transcripción, donde se evalúa dosis, vía, legibilidad, entre otros criterios, instituidas a nivel internacional, adecuados y validado por expertos (tres investigadores responsables del estudio), realizando traducción y retraducción de éste ${ }^{9,10}$.

Se efectuó prueba piloto para evaluar comprensibilidad de las preguntas. Para controlar la calidad del ingreso de datos, se realizó análisis de valores extremos y consistencia entre las preguntas.

En el análisis de la prescripción-trascripción, se consideró como incorrecto, que al menos una acción fuese errada.

Se contó con autorización del Comité de Ética del Servicio de Salud Araucanía Sur y con consentimiento informado de los Jefes de Servicio de las unidades pediátricas.

Los datos fueron ingresados en planilla Excel y procesados por el programa STATA 11.0

Se realizó análisis descriptivo a través de frecuencias, porcentajes, promedios y desviación 
Tabla 1. Acciones de prescripción de medicamentos endovenosos. Servicios pediátricos. H.H.H.A., 2008-2009

\begin{tabular}{|c|c|c|c|c|c|}
\hline Acciones & $\begin{array}{c}\text { Neonatología } \\
\text { n: } 125\end{array}$ & $\begin{array}{l}\text { Pediatría } \\
\text { n: } 125\end{array}$ & $\begin{array}{l}\text { UCI-UTI } \\
\text { n: } 125\end{array}$ & $\begin{array}{l}\text { C. Infantil } \\
\text { n: } 125\end{array}$ & $\mathbf{p}$ \\
\hline Prescripción ilegible & $28(22,4)$ & $14(11,2)$ & $18(14,4)$ & $39(31,2)$ & $<0,001$ \\
\hline No señala dosis & $9(7,2)$ & $7(5,6)$ & $6(4,8)$ & $32(25,6)$ & $<0,001$ \\
\hline No señala vía de administración & $20(16,0)$ & $16(12,8)$ & $28(22,4)$ & $54(43,2)$ & $<0,001$ \\
\hline No señala frecuencia de administración & $13(10,4)$ & $10(8,0)$ & $14(11,2)$ & $37(29,6)$ & $<0,001$ \\
\hline \multicolumn{6}{|l|}{$N^{\circ}$ de acciones incorrectas } \\
\hline 0 & $84(26,8)$ & $99(31,6)$ & $76(24,3)$ & $54(17,3)$ & \\
\hline 1 & $25(23,6)$ & $14(13,2)$ & $39(36,8)$ & $28(26,4)$ & \\
\hline 2 & $7(27,0)$ & $5(19,2)$ & $5(19,2)$ & $9(34,6)$ & \\
\hline 3 & $5(15,2)$ & $5(15,2)$ & $3(9,0)$ & $20(60,6)$ & \\
\hline 4 & $4(18,2)$ & $2(9,1)$ & $2(9,1)$ & $14(63,6)$ & \\
\hline $\begin{array}{l}\text { Presencia de al menos una acción } \\
\text { incorrecta }\end{array}$ & $41(32,8)$ & $26(20,8)$ & $49(39,2)$ & $71(56,8)$ & $<0,001$ \\
\hline
\end{tabular}

Prueba exacta de Fisher.

estándar. Para la comparación de los porcentajes se utilizó la prueba exacta de Fisher y la concordancia entre prescripción y transcripción con la prueba de Mc Nemar. Nivel de significación 5\%.

\section{Resultados}

De las 500 prescripciones estudiadas (125 por cada servicio pediátrico), el equipo de investigación encontró que 19,8\% de estas indicaciones fueron ilegibles, $10,8 \%$ no señaló dosis, $23,6 \%$ de las prescripciones no indicó vía endovenosa como medio de administración y $14,8 \%$ no especificó frecuencia de administración.

En transcripciones, 3,0\% fueron ilegibles, $4,4 \%$ no incluyó la totalidad de los fármacos prescritos y 2,6\% del personal de enfermería transcribió fármacos sin indicación, desde la hoja de indicaciones médicas hacia la hoja de registro de enfermería.

Las prescripciones estudiadas evidenciaron $37,4 \%$ de al menos una acción incorrecta (indicación ilegible, no señalaba dosis, vía o frecuencia de administración), 16,2\% al menos dos, $11,0 \%$ al menos tres y $4,4 \%$ todas las acciones incorrectas.

En transcripciones, 7,8\% presentó al menos una acción incorrecta (transcripción ilegible, no todos los fármacos indicados todos los fármacos indicados transcritos y transcripción de fármacos sin indicación), 2,0\% al menos dos y $0,2 \%$ todas las acciones incorrectas.

Al comparar la presencia de al menos una acción incorrecta en las prescripciones con los servicios pediátricos, se observó diferencias significativas, $\mathrm{p}<0,001$, (Tabla 1 ), similar asociación con las transcripciones, $\mathrm{p}<0,001$, (Tabla 2).

De forma concluyente, se observó relación significativa entre los porcentajes de prescripción y transcripción incorrecta $(\mathrm{p}<0,001)$, entre prescripción y transcripción ilegible $(\mathrm{p}<0,001)$ (Tabla 3).

\section{Discusión y Conclusiones}

El proceso de medicación en prescripción y transcripción se encuentra sujeto a variables físicas, humanas y ambientales, que de no ser mantenidas dentro de estándares de seguridad, pueden constituir una amenaza para el usuario pediátrico. Así, los resultados obtenidos de la investigación, ponen de manifiesto las necesidades de acciones de control y seguimiento en un área de trabajo específica, donde se enmarca la situación de tratamiento de un recién nacido dependiente, inestable y vulnerable. 
Errores en prescripción y transcripción de medicamentos - E. Rivas et al

Tabla 2. Acciones de transcripción de medicamentos endovenosos. Servicios pediátricos. H.H.H.A., 2008-2009

\begin{tabular}{|lrrrrr|}
\hline Acciones & Neonatología & Pediatría & UCI-UTI & C. Infantil & p \\
\hline Transcripción ilegible & $2(1,6)$ & $4(3,2)$ & $6(4,8)$ & $3(2,4)$ & 0,566 \\
\hline No transcripción de fármacos indicados & $1(0,8)$ & $6(4,8)$ & $3(2,4)$ & $12(9,6)$ & 0,005 \\
\hline $\begin{array}{l}\text { Transcripción de fármacos sin indicación } \\
\text { No de acciones incorrectas }\end{array}$ & $1(0,8)$ & $2(1,6)$ & $1(0,8)$ & $9(7,2)$ & 0,006 \\
\hline 0 & $122(26,5)$ & $116(25,2)$ & $117(25,3)$ & $106(23,0)$ \\
\hline 1 & $2(6,9)$ & $7(24,1)$ & $6(20,7)$ & $14(48,3)$ \\
\hline 2 & $1(11,1)$ & $1(11,1)$ & $2(22,2)$ & $5(55,6)$ \\
\hline 3 & $0(0,0)$ & $1(100,0)$ & $0(0,0)$ & $0(0,0)$ \\
\hline $\begin{array}{l}\text { Presencia de al menos una acción } \\
\text { incorrecta }\end{array}$ & $3(7,7)$ & $9(23,1)$ & $8(20,5)$ & $19(48,7)$ & 0,002 \\
\hline
\end{tabular}

Prueba exacta de Fisher.

Tabla 3. Concordancia entre prescripción y transcripción de medicamentos endovenosos. Servicios pediátricos. H.H.H.A., Temuco-Chile 2008-2009

\begin{tabular}{|c|c|c|c|c|c|c|}
\hline \multirow[b]{2}{*}{ Centros } & \multirow[b]{2}{*}{ Prescripción } & \multicolumn{2}{|c|}{ Transcripción } & \multirow{2}{*}{$\begin{array}{c}\% \\
\text { Prescripción } \\
\text { incorrecta }\end{array}$} & \multirow{2}{*}{$\begin{array}{c}\% \\
\text { Transcripción } \\
\text { Incorrecta }\end{array}$} & \multirow[t]{2}{*}{$\mathbf{p}$} \\
\hline & & Incorrecta & Correcta & & & \\
\hline \multirow[t]{2}{*}{ Neonatología } & Incorrecta & 0 & 41 & 32,8 & 2,4 & $<0,001$ \\
\hline & Correcta & 3 & 81 & & & \\
\hline \multirow[t]{2}{*}{ Pediatría } & Incorrecta & 4 & 22 & 10,7 & 7,2 & $<0,001$ \\
\hline & Correcta & 5 & 94 & & & \\
\hline \multirow[t]{2}{*}{ UCI-UTI } & Incorrecta & 4 & 45 & 34,3 & 6,4 & $<0,001$ \\
\hline & Correcta & 4 & 72 & & & \\
\hline \multirow[t]{2}{*}{ C. Infantil } & Incorrecta & 19 & 52 & 52,0 & 15,2 & $<0,001$ \\
\hline & Correcta & 0 & 54 & & & \\
\hline
\end{tabular}

Prueba de Mc Nemar.

En este contexto, la prescripción es el pilar fundamental para que la sucesión de eventos asociados (transcripción, preparación y administración de medicamentos) se ejecute de manera segura, confiable y eficaz.

Resulta complejo determinar la bioseguridad en los procesos evaluados (prescripción -transcripción), si no se cuenta con una actitud profesional objetiva, donde las personas implicadas en el proceso, autoevalúen su accionar y reflexionen respecto de la calidad de los servicios prestados. De igual modo, se requiere desarrollar una cultura de error, donde la evaluación sea dirigida hacia los procesos y el sistema.

En las prescripciones estudiadas, la ilegibilidad presentó un valor importante de asumir (19,8\%), en contraste con estudios de Muñoz et al, el año 2001, quienes muestran una tasa de error de $28,4 \%{ }^{2}$, en estudios similares. Al respecto, la organización de Administración de Medicamentos y Alimentos de los Estados Unidos de Norteamérica (FDA), señala que aproximadamente el $10 \%$ de todos los errores de medicación es resultado de la confusión de nombre de la fármacomedicina ${ }^{11}$. 
Campino y $\mathrm{col}^{8}$ encontraron $35,2 \%$ de error en prescripción, siendo los más frecuentes los de dosificación $(16,4 \%)$ y en el estudio que se presenta la dosificación mostró 10, 8\%.

En transcripción, los resultados arrojaron 7,8\% de error versus $21,3 \%$ de errores obtenidos por Campino y cols ${ }^{8}$.

Respecto a escala de acciones incorrectas en el momento de prescribir medicamentos, se encontró que se cometió al menos un error en 32,8\% (41/125) en el servicio Neonatología (A); 20,8\% (26/125) en el servicio de Pediatría (B); 39,2\% (49/125) en el servicio de UCI-UTI (C) y $56,8 \%$ (71/125) en el servicio de Cirugía Infantil (D). Con p: 0,001 (prueba exacta de Fisher).

En escala de acciones incorrectas, en transcripción, se evidenció que la eventualidad de incurrir en al menos un error fue $7,69 \%(3 / 125)$ servicio A; 23,08\% (9/125) servicio B; 20,51\% (8/125) servicio C y $48,72 \%$ (19/125) servicio D. Con p: 0,002 (prueba exacta de Fisher).

Los errores de medicación son indicadores de calidad en la asistencia sanitaria, por lo que su detección y análisis de causas, pueden ayudar a la prevención sistemática, mejorando así la calidad del proceso asistencial ${ }^{8}$. En este contexto, autores señalan que el uso de un sistema de registro electrónico (ficha electrónica), incrementaría la eficacia del proceso de medicación ${ }^{12}$.

Independiente de las estrategias que se adopten en relación a la mejora del sistema de medicación, entes directivos en salud plantean la necesidad de vigilancia extrema y el desarrollo de proyectos de prevención ${ }^{13}$.

Así, la comunicación eficiente entre los integrantes del equipo sanitario constituiría un potencial para reducir errores de medicación en los usuarios pediátricos ${ }^{14}$, así como también, de reconocer la posibilidad de error, manteniéndose alerta ante situaciones conocidas de alto riesgo, con capacitación constante y personal idóneo, el cual cuente con experticia, sin sobrecarga laboral, vigil y, en lo posible, sin personal part-time ${ }^{15}$.

Las medidas preventivas deben ser evaluadas por un comité de gestión de riesgos ${ }^{16}$, en hospitales y servicios sanitarios, desde la dispensación de los medicamentos, por parte de los químicos farmacéuticos, hasta la administración por parte del equipo de enfermería, teniendo en consideración que existe consenso a nivel internacional en aceptar la regla emitida por Leape en $1994^{17}$, a través de la cual, se deben visualizar estrategias de prevención de errores, centradas en el propio sistema y no en el individuo ${ }^{18}$, lo que exige paralelamente una apertura de mente y cambio de paradigmas.

Por otra parte, existe una responsabilidad moral de realizar investigación-formación y docencia, proporcionando datos para el análisis estadístico que evidencie científicamente y que permita la mejora continua de las prestaciones realizadas. Los profesionales sanitarios deben promover protocolos, informes estadísticos, documentación asistencial y administrativa que guarden relación con los procesos clínicos de la medicación ${ }^{7}$.

\section{Referencias}

1. Kozer E, Scolnik D, Macpherson A, Rauchwerger D, Gideon Using a Preprinted Order Sheet to Reduce Prescription Errors in a Pediatric Pediatrics 2005; 116: 1299-302.

2. Muñoz M, Pallás C, De La Cruz J, López M, Moral A, Balaustegui A. Errores en las prescripciones médicas en una unidad neonatal. An Esp Pediatr 2001; 55: 535-40.

3. Grespan V, D'Innocenzo M. Evaluation of the quality of nursing documentation though the review of patient medical records. Acta Paulista Enfermagen 2009; 22: 317-7.

4. Otero M, Martín R, Robles M, Codina C. Errores de medicación. Farmacia Hospitalaria. p. 742. Disponible en: http://www.ismp-espana.org/publicaciones/ [citado en enero 4 de 2010].

5. The National Coordinating Council for Medication Error Reporting and Prevention (NCC MERP): The First Ten Years "Defining the Problem and Developing Solutions”, 2005 www.nccmerp.org [citado en octubre 9 de 2009].

6. Booth B. Management of drug errors. Nurs Times 1994; 90: 30-31.

7. Ruiz Hontangas A. Calidad en los Registros de Enfermería, 2005. Disponible en http://www.fac.org.ar/ccvc/ llave/c028/ruizhont.php [citado en enero 4 de 2010].

8. Campino Villegas M, López Herrera M, García Franco I, López de Heredia Y, Goya A, Valls I Soler. Errores en la prescripción y trascripción de medicación en una unidad neonatal. Bilbao. España, 2006. Disponible en: http://www.ncbi.nlm.nih.gov/pubmed/16606569 [citado en enero 4 de 2010].

9. American Academy Of Pediatrics. Prevention of medication errors in the pediatrics inpatient setting. Pediatrics 2003; 112: 431-6. 
Errores en prescripción y transcripción de medicamentos - E. Rivas et al

10. American Society Of Health-System Pharmacist (ASHP). Guidelines on preventing medication errors in hospitals. Am J Health-Syst Pharm 1993; 50: 305-14.

11. Rados C. Confusión de Nombre de Medicina (Droga): Prevención de Errores de Medicación. FDA Consum. 2005; 39: 35-7.

12. Nelson N, Evans R, Samore M, Gardner R. Detection and Prevention of Medication Errors Using Real-Time Bedside Nurse Charting. J Es Med 2005; 12: 390-7.

13. Gray J, Goldmann D. Medication errors in the neonatal intesive care unit: Special patients, unique issues. Arch Dis Child Fetal Neonatal 2004; 89: 472-3.

14. Fortescue E, Causal R, Landrigan C, Mckenna K, Clapp $\mathrm{M}$, Federico F, et al. Prioritizing Strategies for Preventing Medication Errors and Adverse Drug Events in Pediatric
Inpatients. Pediatrics 2003; 111: 722-9.

15. Campaña G. Surgical adverse events. Prevention. Chapter II. Medication errors in the operating room. Rev Chil Cir 2006; 58: 305-7.

16. Beyea S, Hicks R, Becker S. Medication Errors in the OR. A Secondary Analysis of Medmarx. AORN. Journal 2003; 77: 122-34.

17. Leape LL. Error in medicine. JAMA 1994; 272: 1851-7.

18. Pasto-Cardona L, Masuet-Aumatell C, Bara-Oliván B, Castro-Cels I, Clopés-Estela A, Páez-Vives F, et al. Estudio de incidencia de los errores de medicación en los procesos de utilización del medicamento: prescripción, transcripción, validación, preparación, dispensación y administración en el ámbito hospitalario. Farm Hosp 2009; 33: 257-68. 